\title{
EVENTRATION OF DIAPHRAGM COMPLICATED BY GASTRIC VOLVULUS IN ELDERLY FEMALE: A CASE REPORT
}

\author{
Kedarnath Arya ${ }^{1}$, S. V. Sahu², R. Gautam ${ }^{3}$, Ashok Kumar', R. Sinha ${ }^{5}$
}

\section{HOW TO CITE THIS ARTICLE:}

Kedarnath Arya, S. V. Sahu, R. Gautam, Ashok Kumar, R. Sinha. "Eventration of Diaphragm Complicated by Gastric Volvulus in Elderly Female: A Case Report". Journal of Evolution of Medical and Dental Sciences 2014; Vol. 3, Issue 14, April 07; Page: 3600-3604, DOI: 10.14260/jemds/2014/2327

\begin{abstract}
We report a 64 year old female who presented with epigastric pain and intractable vomiting not respond to treatment and we were not able to pass nasogastric tube. Chest $\mathrm{x}$-ray shows eventration of left sided hemi diaphragm, upper gastro intestinal contrast study conform the diagnosis of gastric volvulus. Plication of left hemidiaphragm with anterior gastropexy was performed through an abdominal approach. Postoperatively the patient's symptoms improved. Acute gastric volvulus carries a mortality rate of $42-56 \%$, secondary to gastric ischemia, perforation or necrosis. ${ }^{1}$ Emergency physicians should have suspicion about gastric volvulus when treating patients with abdominal pain and persistent vomiting. The patient should go for surgical consultation as early as possible.
\end{abstract}

KEYWORDS: Eventuation diaphragm, gastric volvulus, elderly female.

INTRODUCTION: In diaphragmatic eventration, permanent elevation of an immobile hemidiaphragm occurs in which peripheral muscular attachment is normal with no interruption in peritoneal or pleural layers. It usually remains asymptomatic in early life and presents later with respiratory and occasionally gastrointestinal complications. This may lead to displacement of abdominal organs, especially the stomach. Gastric volvulus is an uncommon complication of eventration of diphragm. ${ }^{2}$

There are two types of gastric volvulus: organoaxial and mesenteroaxial. The most common type is organoaxial, in which the stomach rotates along the longitudinal axis and is associated with paraesophageal hernias. The mesenteroaxial type, in which the stomach rotates between the lesser and greater curvatures, is believed to be idiopathic, causing chronic symptoms. ${ }^{3}$

The presence of persistent vomiting and epigastric pain despite initial antiemetic treatment should trigger one to think of gastric volvulus, despite the patient appearing very stable and healthy. Diagnosis of gastric volvulus, which can have significant morbidity and mortality can be easily missed. Early radiological imaging with x-ray or computed tomography (CT) can facilitate the management of the patient.

Eventration of diaphragm is defined as an abnormal elevation of an intact diaphragm and most often is characterized by a developmental abnormality of the diaphragm musculature. ${ }^{4}$ It usually remains asymptomatic in early life and presents later with respiratory and occasionally gastrointestinal complications.

The abnormally wide diaphragmatic space provides the potential for abnormal rotation of stomach around itself. This abnormal rotation is known as gastric volvulus. It is of two varieties. One is organoaxial (more common) and other is mesentero-axial. We present a case of 60 year old female with eventration of left hemidiaphragm with chronic intermittent organo-axial gastric volvulus. 


\section{CASE REPORT}

CASE REPORT: A64 year old female presented to us as intermittent vomiting, epigastric pain and increase gas formation, not able to tolerate sips of water. Patient was put nil per oral and I. V fluid support started. We were not able to pass nasogastric tube. On physical examination, patient was alert well oriented to time place person, her vitals were normal except tachycardia and tachypnea.

There was epigastric fullness and tenderness on deep palpation. Her routine investigation (Hb\%, TLC, DLC, ESR) including serum sodium and potassium were normal. X-ray chest shows left sided elevation of diaphragm with large gaseous shadow and air fluid level in left side chest

[Figure 1]. On upper G I endoscopy endoscope cannot be negotiated beyond pylorus. An upper GI barium study was preferred over CT to look for the distended hollow viscus and the elevated hemidiaphragm. The upper GI Barium study showed widely distended stomach lying in horizontal plane with the gastroesophageal junction at an upper level than pylorus. These findings were consistent with organoaxial type of gastric volvulus with eventration of left hemi-diaphragm.

[Figure 2]. Patient was planned for surgery; a left subcostal incision was given to asses left diaphragm. On exploration eventration of left side of diaphragm, dilated stomach and transverse colon were present in it [figure3]. Adhesions of stomach and colon with diaphragm was cut and anterior gastropexy was done.The diaphragmatic plication followed by meshplasty and left sided chest tube drainage was done.

Post-operative period remain un eventful and chest x-ray shows complete chest expansion [Figure 4]. Patient was discharged on 6th post-operative day. The patient was last seen at 7 month follow up and has progressed well.

DISCUSSION: Eventration is a congenital anomaly of the diaphragm characterized by muscular aplasia and subsequent abnormal elevation of an intact hemidiaphragm. The left side is affected more than the right, and male are affected more often than female. The phrenic nerve-appear normal, but the eventrated leaf can consist of a facial layer with few or no muscle fibre between the pleura and peritoneum. Gastric volvulus is a potentially life-threatening entity and most cases of gastric volvulus occur in association with eventration of left hemidiaphragm or a hiatal hernia ${ }^{5}$ Since 1897, over 400 cases have been reported in literature; at least $2 / 3^{\text {rd }}$ are of chronic or recurring type. 6

The stomach is relatively fixed at the esophageal hiatus and pylorus and is prevented from abnormal rotation by 4 gastric ligaments. Absence or weakness of these anatomic anchors results in the mobility of the stomach within the wide subdiaphragmatic space and potential for gastric volvulus. ${ }^{7}$ Organoaxial rotation is the most common type accounting for greater than $60 \%$ of the cases, followed by mesenteroaxial. A combined organo-axial and mesenteroaxial rotation is the least common type. Primary gastric volvulus, making up one third of cases, occurs when the stabilizing ligaments are too lax as a result of congenital or acquired causes. Secondary gastric volvulus, making up the remainder of cases, occurs in association with a paraesophageal hernia or other congenital or acquired diaphragmatic defects. ${ }^{8}$

Patients with unilateral diaphragmatic eventration are asymptomatic, however, some complain of dyspnea on effort or rarely orthopnea, due to the decrease in ventilation and oxygenation because of paradoxical motion of the affected diaphragm during inspiration and expiration. The severity of either symptom depends on presence or absence of underlying pulmonary disease.

Gastrointestinal (GI) symptoms may even predominate when related to volvulus of the stomach with intermittent or complete outlet obstruction. ${ }^{4}$ Symptoms of diaphragmatic eventration 
may include nausea, heartburn, early satiety, post postprandial vomiting, constipation and epigastric discomfort.

The classic triad of retching, severe and constant epigastric pain, and difficulty in passing a nasogastric tube should suggest the presence of acute gastric volvulus. ${ }^{9}$ The diagnosis of diaphragmatic eventration associated with gastric volvulus is usually straight forward and can be established by history and, in most cases, by routine chest X-rays and either upper GI series or CT scan. Management of diaphragmatic eventration varies rarely depending on whether the diagnosis is made in infants or adults. Simple cases of diaphragmatic eventration may not require surgical intervention if it is not intruding significantly into the thoracic cavity and is not associated with adverse symptoms.

However, symptomatic gastric volvulus associated with diaphragmatic eventration is a surgical emergency and always requires surgical intervention ${ }^{5}$. Treatment is conservative or surgery.

The risk of gastric perforation is significant in conservative treatment. Therefore, patients should be considered carefully for conservative treatment. The gold standard is open laparotomy with detorsion and reversion with anterior gastropexy. Gastric volvulus is an uncommon cause of upper abdominal pain and persistent vomiting.

This diagnosis must be suspected in patients with documented diaphragm eventration. The presence of persistent vomiting despite initial antiemetic treatment, continuing epigastric pain and inability to pass a nasogastric tube should trigger one to think of gastric volvulus. Recently endoscopic treatment is evolving.

\section{REFERENCES:}

1. Chau B, Dufel S. Gastric volvulus. Emerg Med J 2007; 24: 446-7.

2. Kim HS, Yoo JS, Han SJ, Park H. Chronic recurrent volvulus of the colonic splenic flexure associated with the eventration of left diaphragm. Korean J Gastroenterol 2007; 49(1):37-40.

3. Moy RK, Salazar AM, Chan SB. Inability to pass a nasogastric tube: a surgical emergency. Am J Emerg Med 2007; 25: 213-5.

4. Wayne ER, Campbell JB, Burrington JD, Davis WS. Eventration of the diaphragm. J Pediatr Surg.1974;9: 643-651. doi: 10.1016/0022-3468(74)90101- .[PubMed] [Cross Ref.]

5. Shah NN, Mohsin M, Khursheed SQ, Farooq SS, Buchh AA, Quraishi AQ. Eventration of diaphragm with gastric volvulus: a case report. Cases J 2008; 1: 404.

6. Camblos JFB. Acute volvulus of stomach. Am Surg.1969;35: 505-509.

7. .Par W, Choi S, Suh S. Pediatric gastric volvulus - Experience with 7 cases. J Korean Med Sci. 1992; 7: 258-263. [PMC free article] [PubMed].

8. Lee JS, Park JW, Sohn JW, Kim KC, Hwang SG, Park PW, et al. Organo-axial volvulus of the stomach with diaphragmatic eventration. Korean J Intern Med.2000; 15:127-130. [PubMed].

9. Llaneza PP, Salt WB. 2nd Gastric volvulus. More common than previously thought? Postgrad Med. 1986; 80: 279- 83. [PubMed] 


\section{CASE REPORT}

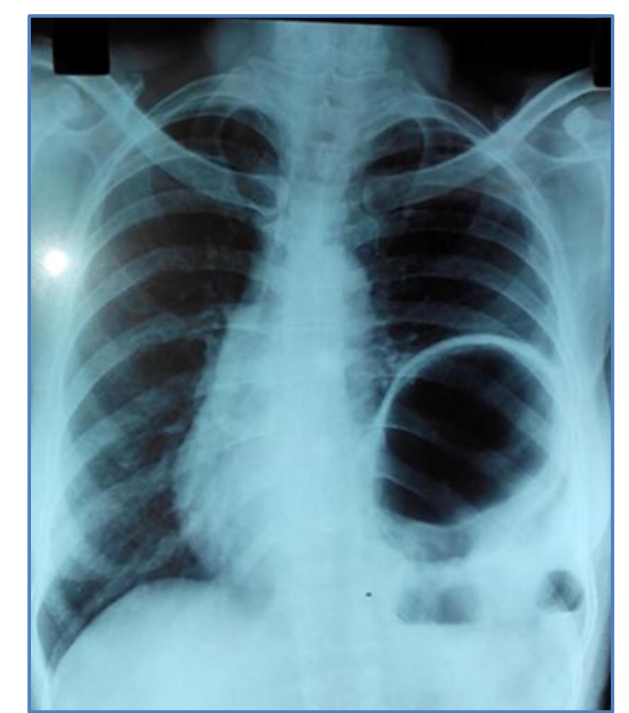

Fig. 1: Pre- Operative x-ray chest

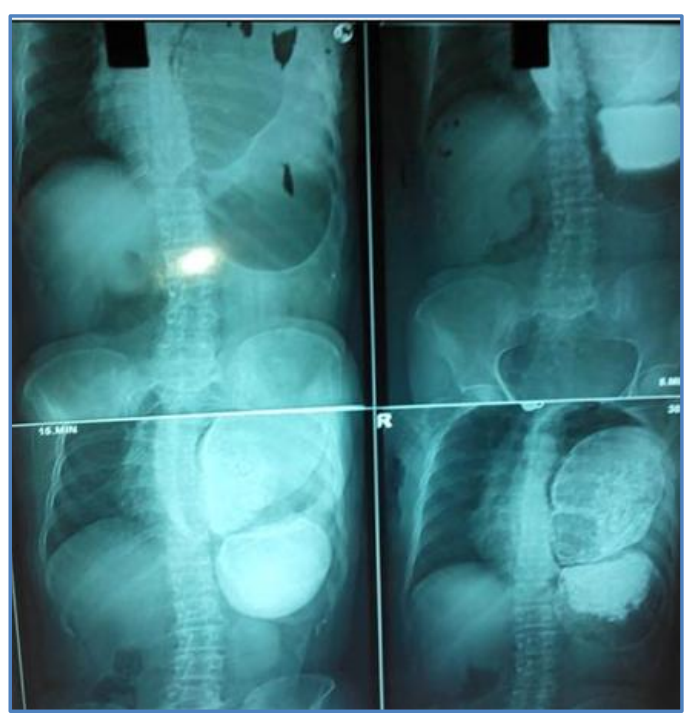

Fig. 2: Barium constrast study showing Gastric Volvulus and Eventration of Left diaphragm

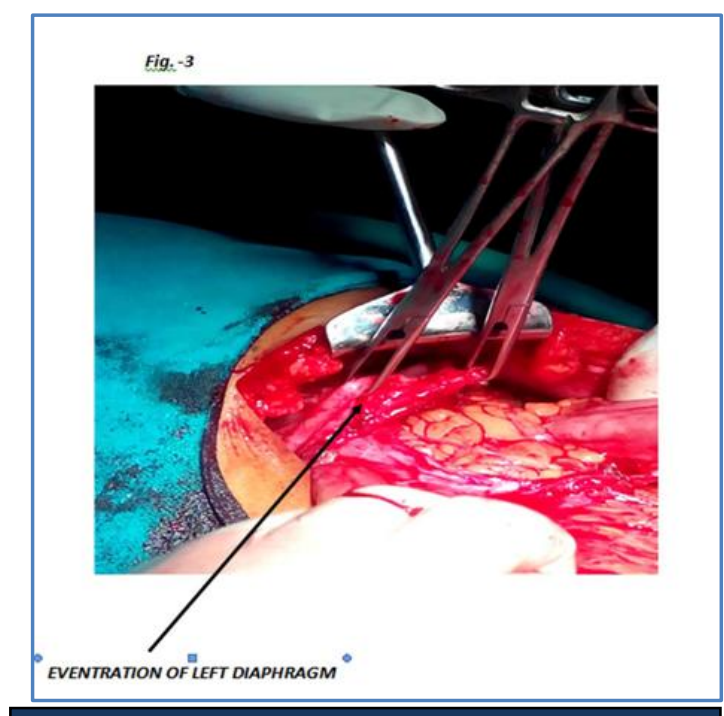

Fig. 3: Eventration of Left Diaphragm

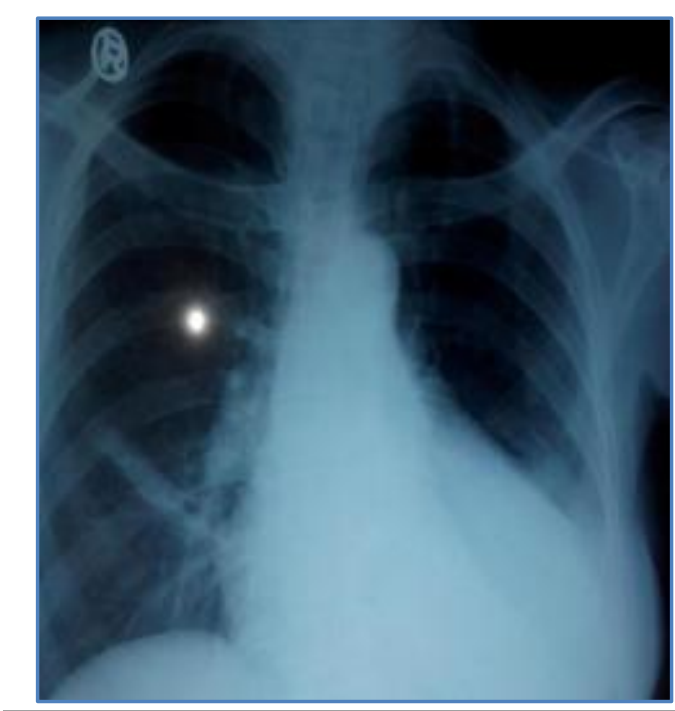

Fig. 4: Post- Operative $x$-ray chest 


\section{CASE REPORT}

\section{AUTHORS:}

1. Kedarnath Arya

2. S. V.Sahu

3. R. Gautam

4. Ashok Kumar

5. R. Sinha

\section{PARTICULARS OF CONTRIBUTORS:}

1. Assistant Professor, Department of General Surgery, MLB Medical College, Jhansi, U. P.

2. Senior Resident, Department of General Surgery, MLB Medical College, Jhansi, U. P.

3. Assistant Professor, Department of General Surgery, MLB Medical College, Jhansi, U. P.

4. Junior Resident, Department of General Surgery, MLB Medical College, Jhansi, U. P.
5. Professor and Head, Department of Surgery, MLB Medical College, Jhansi, U. P.

\section{NAME ADDRESS EMAIL ID OF THE CORRESPONDING AUTHOR:}

Dr. K. N. Arya,

DM - 2/9, Veerangana Nagar,

Jhansi, U. P.

E-mail: kedarnath_arya@rediffmail.com

Date of Submission: 11/03/2014. Date of Peer Review: 12/03/2014.

Date of Acceptance: 20/03/2014.

Date of Publishing: 01/04/2014. 